\title{
ORIGINAL ARTICLE \\ Reproductive interference and fecundity affect competitive interactions of sibling species with low mating barriers: experimental and theoretical evidence
}

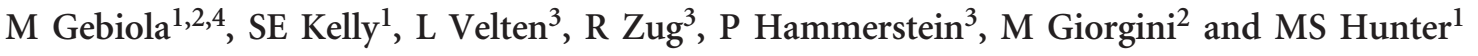 \\ When allopatric species with incomplete prezygotic isolation come into secondary contact, the outcome of their interaction is not \\ easily predicted. The parasitoid wasp Encarsia suzannae (iES), infected by Cardinium inducing cytoplasmic incompatibility (Cl), \\ and its sibling species $E$. gennaroi $(E G)$, not infected by bacterial endosymbionts, may have diverged because of the \\ complementary action of $\mathrm{Cl}$ and asymmetric hybrid incompatibilities. Whereas postzygotic isolation is now complete because of \\ sterility of $\mathrm{F} 1$ hybrid progeny, prezygotic isolation is still incipient. We set up laboratory population cage experiments to evaluate \\ the outcome of the interaction between ES and EG in two pairwise combinations: iES vS EG and cured ES (cES, where \\ Cardinium was removed with antibiotics) vs EG. We also built a theoretical model aimed at exploring the role of life-history \\ differences and asymmetric mating on competitive outcomes. In three of four cages in each treatment, ES dominated the \\ interaction. We found evidence for reproductive interference, driven by asymmetric mating preferences, that gave a competitive \\ edge to $\mathrm{ES}$, the species that better discriminated against heterospecifics. However, we did not find the fecundity cost previously \\ shown to be associated with Cardinium infection in iES. The model largely supported the experimental results. The finding of \\ only a slight competitive edge of ES over EG in population cages suggests that in a more heterogeneous environment the species \\ could coexist. This is supported by evidence that the two species coexist in sympatry, where preliminary data suggest \\ reproductive character displacement may have reinforced postzygotic isolation.
}

Heredity (2017) 119, 438-446; doi:10.1038/hdy.2017.56; published online 13 September 2017

\section{INTRODUCTION}

Reproductive interactions between incipient species are likely to play an important role in shaping their evolutionary trajectories. An important step in the speciation process is the formation of behavioural mechanisms that reinforce reproductive isolation between populations (Ord et al., 2011). When populations that have diverged in allopatry come into secondary contact, selection to minimize competition can accentuate ecological differences between them (Coyne and Orr, 2004). If these populations interbreed and produce hybrids of low fitness, then reinforcement can finalize speciation by promoting the evolution of complete prezygotic isolation (Liou and Price, 1994; Servedio and Noor, 2003). Different types of isolation barriers have ecological consequences that may affect the outcome of interspecific competition and the likelihood of coexistence that in turn will influence gene flow when isolation is incomplete.

Relevant to the current study, a type of reproductive incompatibility in arthropods caused by inherited bacterial symbionts, cytoplasmic incompatibility (CI), can serve simultaneously as an isolating factor (Bordenstein, 2003) and one likely to influence competitive outcomes between incipient species. When present in just one of two populations, CI bacterial symbionts may cause a type of embryo inviability called unidirectional CI. Few or no offspring are produced when symbiont-infected males from one population mate with uninfected females from the other (the CI cross) (Werren et al., 2008). CI symbionts can play a role in competitive outcomes in two ways, with opposite effects. In the first role, they can benefit the infected population by depressing the reproduction of females of the uninfected population when males from the infected population mate with them; this effect differentially influences the uninfected population if hybrids produced in the other crossing direction are viable and fertile. Second, hosts with CI symbionts may bear a fecundity cost (Egas et al., 2002; Perrot-Minnot et al., 2002; de Almeida et al., 2011); this then can reduce the chances of the infected population prevailing in competition with the uninfected population. The strength of these two effects is likely to vary in each experimental system.

Other isolating factors may also influence competitive outcomes. Reproductive interference, defined as an interspecific sexual interaction that decrease the fitness of at least one of the species involved, may tip the outcome of competition towards prevalence of the species least affected (Gröning and Hochkirch, 2008; Kyogoku, 2015). Reproductive interference occurs mainly when allopatric species come into secondary contact after speciation, reinforcing differences in reproductive biology that are already in place, or selecting for changes in mating traits that lead to divergence in their species recognition

${ }^{1}$ Department of Entomology, University of Arizona, Tucson, AZ, USA; ${ }^{2}$ CNR-Istituto per la Protezione Sostenibile delle Piante, Portici, Italy and ${ }^{3}$ Institute for Theoretical Biology, Humboldt-Universität zu Berlin, Berlin, Germany

Correspondence: Dr M Gebiola, Department of Entomology, University of California, Riverside, 3401 Watkins Drive, Riverside, CA 92521, USA.

E-mail: marco.gebiola@gmail.com

${ }^{4}$ Current address: Department of Entomology, University of California, Riverside, Riverside, CA, USA.

Received 11 April 2017; revised 8 August 2017; accepted 9 August 2017; published online 13 September 2017 
systems (that is, reproductive character displacement) (Cothran, 2015). Reproductive interference is expected to be more common in closely related species because of the similarity of their reproductive biology, and has been seen when an alien invasive species interacts with a closely related species in the invaded geographic area (Liu et al., 2007; Bargielowski et al., 2013).

Encarsia gennaroi and Encarsia suzannae (Hymenoptera: Aphelinidae) are parasitoid wasps of whiteflies. They are sibling species that are differentiated morphologically, genetically and by status of infection by bacterial endosymbionts. Encarsia suzannae (iES) is infected by a CIinducing strain of the symbiont Cardinium (Hunter et al., 2003; Gebiola et al., 2017), whereas E. gennaroi (EG) is not infected. These two species are allopatric in part of their ranges; uninfected E. gennaroi is common in California and has been recorded in Florida and Texas, whereas infected E. suzannae has been ever recorded only in the lower Rio Grande Valley of Southern Texas (Gebiola et al., 2017). Here, however, the two species have been recorded in sympatry from the early 1990s to the present (Johnson, 1996; Gebiola et al., 2017). Crossing experiments between a population of EG from the region of allopatry and a population of $\mathrm{iES}$ from the region of sympatry (the only area where it is known to occur), and between EG and cured strain of E. suzannae (cES, where Cardinium was removed with antibiotics), showed that speciation in this system was potentially influenced by two complementary isolating mechanisms: CI and asymmetric hybrid incompatibilities (Gebiola et al., 2016). In the present study, we used these same populations that still easily hybridize because of incomplete prezygotic isolation (assortative mating in choice mating tests, but no discrimination in no choice tests), despite complete postzygotic isolation (F1 hybrid females are sterile) (Gebiola et al., 2016). This system is ideal to simulate a secondary contact scenario with multigenerational population cage laboratory experiments, in part because of the small size $(\sim 1 \mathrm{~mm})$ and short generation time ( $\sim 3$ weeks) of the parasitoid wasp hosts. To our knowledge, no other study has explicitly addressed how CI affects reproductive interference and competitive outcomes in such a scenario.

Because postzygotic isolation is complete in this system, the scope for CI to play a direct role in competitive interactions between the species is limited. Gebiola et al. (2016) showed that laboratory crosses between EG and $\mathrm{iES}$ result in $\sim 70 \%$ offspring mortality in each direction, by mainly $\mathrm{CI}$ in one direction and by genetic incompatibility in the other. In contrast, in the crosses in which Cardinium is absent $(\mathrm{EG} \times \mathrm{cES})$ many more hybrids were produced in the direction $\mathrm{EG}$ female $\times$ cES males ( $\sim 80 \%$ F1 hybrids are viable) than in the opposite direction $(\sim 30 \%)$. However, all of the F1 female hybrids produced in all crosses are sterile. Removing the CI Cardinium symbiont thus has little functional effect, in that it exchanges the dead embryos caused by CI with adult females that cannot reproduce. Furthermore, although the abundant sterile hybrids produced in the EG female $\times$ cES male cross could be significant if these hybrid individuals interfere with mating of fertile wasps of the parental species, interference in this haplodiploid system is unlikely because (1) only the F1 females are hybrids (F1 males bear half of the maternal genome only, and hence are not hybrids) and (2) males of both species are polygamous, and hence males that mate with sterile hybrid females will have other mating opportunities.

There would appear to be more potential for an indirect effect of CI symbiont infection, through fecundity cost, to be important in this system. Perlman et al. (2008) showed that there is a relatively high cost associated with Cardinium infection in E. suzannae (= Encarsia pergandiella in that paper). Therefore, if the species' life histories are otherwise very similar, as it might be expected for sibling species, one would predict the uninfected population to benefit.

Finally, Gebiola et al. (2016) recorded significant asymmetric mating preferences in female choice mating tests in this system. Infected and cured ES females (from the area of sympatry) discriminated against EG males more than the EG females (from the area of allopatry) discriminated against ES males in the reciprocal cross. The cost of heterospecific mating should be particularly high if, as in the current system, postmating isolation is complete and females mate only once, or are willing to mate again only after sperm depletion. Reproductive interference is commonly asymmetric in nature, as it is unlikely that two species have completely similar reproductive behaviours (Hochkirch et al., 2007).

For these reasons, we tested the interactions of EG vs iES and EG vs cES in replicated population cages. We expected that (1) we would not find evidence of a direct effect of CI on competition, given reciprocal hybrid sterility and male polygamy; (2) the CI symbiont would impose a fecundity cost that would decrease the relative competiveness of Cardinium-infected $\mathrm{iES}$, reducing the dominance of $\mathrm{iES}$ in the EG vs iES treatment; (3) asymmetric female mating preferences would influence the outcome of the competition, with ES benefitting. From these expectations, we could not predict the outcome of the interaction between EG and iES as the better discrimination by iES females against heterospecific males should be counterbalanced by the fecundity cost associated with symbiont infection that they suffer. On the other hand, the competition between EG and cES should result in cES having a competitive edge over EG because this species discriminates better against heterospecific males and should not suffer a fecundity cost.

\section{MATERIALS AND METHODS}

\section{Study system}

E. suzannae and E. gennaroi are solitary hymenopteran parasitoids of whiteflies with an unusual 'autoparasitic' biology (Hunter and Woolley, 2001). Females are primary parasitoids and lay single female eggs in whitefly nymphs, whereas male eggs are laid in developing wasp larvae or pupae of their own species or other primary parasitoids enclosed within the whitefly cuticle. These sibling species are genetically and morphologically distinct. The most obvious external difference is the colour and pigment pattern: E. suzannae is entirely yellow, whereas E. gennaroi has a partly brown thorax and abdomen (Gebiola et al., 2017). The Cardinium-infected E. suzannae culture (hereafter iES) was established from samples collected from Bemisia tabaci in the Rio Grande Valley, Texas, in 2003, where E. suzannae lives in sympatry with EG, and has been maintained in the laboratory on B. tabaci reared on cowpea plants (Vigna unguiculata) ever since. This culture is fixed for Cardinium infection (Hunter et al., 2003), and the infection is strictly maternally transmitted (Perlman et al., 2008). The cured E. suzannae line (hereafter cES) was obtained by feeding infected adult wasp antibiotics $\left(50 \mathrm{mg} \mathrm{ml}^{-1}\right.$ rifampicin in honey for three generations). The E. gennaroi culture (hereafter EG) originated from a population strictly allopatric relatively to E. suzannae, the latter being never recorded outside of Southern Texas (Gebiola et al., 2017). In particular, EG originated from samples collected in Portici, Italy, where this North American species was first introduced from California in 1979 for classical biological control of Trialeurodes vaporariorum (Gebiola et al., 2017). The three cultures (cES, iES and EG) were reared in environmental chambers at $27^{\circ} \mathrm{C}$ in the Hunter laboratory at the University of Arizona, Tucson, AZ, USA, on B. tabaci (the host for female development) feeding on cowpeas, whereas the host for male development was a primary parasitoid, Eretmocerus emiratus for iES and cES, and Encarsia formosa for EG.

\section{Population cages}

To determine the influence of interspecific $\mathrm{CI}$ on the outcome of competition, the population cages were set up in two treatments, one in which the 
uninfected EG competed with iES, and the other in which EG competed with cES. Each cage, four per treatment, consisted of a $50 \mathrm{~cm}^{3}$ ventilated, plexiglass cage housing plants bearing hosts for the parasitoid wasps. At the start of the experiment the two wasp species were introduced at equal frequency. After parasitism of hosts in each subsequent generation, all of the leaves bearing wasp pupae were removed, placed in an emergence jar, and emerging adult wasps were used to start the subsequent generation as well as to sample for the relative frequency of parental and hybrid wasps. Because both of these Encarsia species are autoparasitic, three plants bearing whitefly hosts for female eggs and two plants bearing parasitoid pupae of $E$. formosa for male eggs were provided in the cages at each generation. The majority of whiteflies were first- or second-instar nymphs at the time those plants were introduced to the cages, whereas the majority of the parasitoid hosts, E. formosa, were early pupae. The initial introduction of EG and iES or cES in each cage consisted of 80 virgin females and 40 male adult wasps of each species, collected in modified Pasteur pipettes in groups of 20 wasps of the same species and sex per pipette. The pipettes were regularly arrayed among plants before wasp release to ensure equal opportunities for intraspecific and interspecific mating and host finding. The eight cages were placed on two shelves inside a climatic chamber $\left(27^{\circ} \mathrm{C}, 16 \mathrm{~h} \mathrm{light} / 8 \mathrm{~h}\right.$ dark and ambient humidity). Plants bearing $E$. formosa were removed from the cages 2 days after the introduction of wasps to avoid the emergence of E. formosa adult females inside the cages, and the leaves were placed in an emergence jar. To reduce the risk of the remaining live adult ES or EG females hyperparasitizing wasp pupae of either species and potentially influencing competitive outcomes, the plants bearing whiteflies were fumigated for $2 \mathrm{~h}$ with a dichlorvos 'No Pest Strip' 7 days after wasp introduction. This treatment kills adult wasps but has no influence on whitefly nymphs or pupal wasps (Perlman et al., 2008). After 4 days, before wasps of either species emerged, leaves from plants of each cage were harvested and placed in an emergence jar. These jars contained leaves bearing all pupae of the two parental species and hybrid wasps. At 22 days after the first wasp introduction, at about the midpoint of adult wasp emergence, a subset of 80 females and 40 males from the first generation were randomly aspirated from different areas of the emergence jars, and reintroduced to the cage to start the new generation. This sampling was effectively 'blind' because the wasp species cannot be distinguished by the naked eye due to their small size and similar colour. All remaining wasps were then aspirated from each jar, and stored in $95 \%$ ethanol at $-20^{\circ} \mathrm{C}$ for subsequent molecular analyses. The experiment was run for five discrete generations, with each generation of each cage initiated with wasps randomly sampled from the previous generation as described directly above. At generation F3 there was a shortage of females and males in cages 3 (treatment EG vs iES), 5 and 7 (treatment EG vs cES) because of the poor health of plants, and hence only 60 $+40,40+20$ and $15+10$ females and males were introduced to start F4 in these cages, respectively.

\section{High-resolution melting}

Although the sibling species EG and ES differ in colouration, and hybrid wasps have a colour that is intermediate between those of the parental species, a large proportion of hybrids and some adults of the two species were not clearly distinguishable. Therefore, in order to unambiguously assign individuals to hybrid, EG and ES categories, we developed a high-resolution melting (HRM) assay. This technique is based on the fact that even small DNA sequence differences lead to different melting temperatures. The melting protocol is applied following amplification of short quantitative PCR (qPCR) products, where the differences in melting curves can be used to assign identity to individuals (Reed et al., 2007). To develop the HRM protocol, we sequenced the single-copy nuclear gene elongation factor 1- $\alpha$ for both parental species and known hybrids, and identified a portion of the gene that contained two singlenucleotide polymorphisms that were homozygous for the parental species and heterozygous for the hybrids. We then used the Primer3 online software (Untergasser et al., 2012) to design primers that could amplify a short fragment including the two diagnostic single-nucleotide polymorphisms. We chose the following primers: HRMF: $5^{\prime}$-GATCAGTAACGGCTACACTCCA- 3 ', and HRMR: ${ }^{\prime}$-CGCACTTCTCCTTGATCTCG- ${ }^{\prime}$. The primers amplified a short $83 \mathrm{bp}$ fragment that showed clear differences in the melting curves of each parental type as well as the hybrids, as predicted by uMelt (Dwight et al., 2011).
The primers were then tested to check for secondary structure using the DINAmelt Server (available at: http://unafold.rna.albany.edu/?q= DINAMelt/ Two-state-folding), then for specificity and for primer dimers by running a PCR and examining the amplicons on an agarose gel.

After a trial-and-error process in which we evaluated a number of DNA isolation protocols (chelex and kit column extractions primarily) for their compatibility with HRM, we used a 'direct PCR' approach that involved no DNA isolation. We experimented with the use of intact insects in PCR-grade water within the reaction tubes (Wong et al., 2014; Rugman-Jones and Stouthamer, 2016). However, because fluorescence in the Bio-Rad (Hercules, CA, USA) CFX-connect qPCR machine that we used is measured by an optical pathway that travels through the length of the reaction tube, the placement of the insect, although small, interfered with the fluorescent signal from the intercalating dye, and hence we had to grind the wasps. For each cage of each generation, we randomly selected 24 females among those stored in ethanol at the end of each generation by pipetting without observation. These wasps were ground individually in $10 \mu \mathrm{l}$ of PCR-grade water using a $20 \mu$ l beveled pipette tip on a Parafilm strip under a stereomicroscope, and the homogenate was put on ice until use. Then, $8 \mu \mathrm{l}$ water containing the ground wasp was added to a $20 \mu \mathrm{l}$ PCR mix, containing $0.15 \mu \mathrm{l}$ of Taq polymerase (New England Biolabs, Ipswich, MA, USA) and $2 \mu \mathrm{l}$ of $10 \times$ buffer (New England Biolabs), $1.2 \mu \mathrm{l}$ of $10 \mathrm{~mm}$ dNTPs, $1.2 \mu \mathrm{l}$ of $5 \mu \mathrm{m}$ primers and $1 \mu \mathrm{l}$ of $20 \times$ EvaGreen (Biotium, Fremont, CA, USA). Both PCR amplification of the target sequence and sequence melting were performed in a Bio-Rad CFX-connect qPCR machine, and four cages were screened in a 96-well qPCR plate. PCR was performed with the following settings: $2 \mathrm{~min}$ initial denaturation at $95^{\circ} \mathrm{C}$, followed by 40 cycles of $5 \mathrm{~s}$ denaturation at $95^{\circ} \mathrm{C}$ and $10 \mathrm{~s}$ annealing at $60^{\circ} \mathrm{C}$, with a $3 \mathrm{~min}$ hold at $72^{\circ} \mathrm{C}$ at the end to allow complete amplicon extension. Before the melt, the PCR products were exposed to $95^{\circ} \mathrm{C}$ for $30 \mathrm{~s}$ followed by $30 \mathrm{~s}$ at $55^{\circ} \mathrm{C}$ to insure that all products reassociated and to encourage heteroduplex formation. The HRM phase consisted of a melt domain span of $12^{\circ} \mathrm{C}$ (from $77^{\circ} \mathrm{C}$ to $89^{\circ} \mathrm{C}$ ), with increases of $0.2^{\circ} \mathrm{C}$ over $10 \mathrm{~s}$, an instrument setting recommended for Bio-Rad CFX96 by Li et al. (2014), with each temperature increase followed by a plate read of the fluorescence signal. Melting curves were then checked using the Bio-Rad CFX Manager Software, and in most instances could be easily assigned to each of the three groups. In the very few cases where curves were ambiguous, the custom MW6.2 software written in LabView (National Instruments, Austin, TX, USA) was used with the available output data to unambiguously assign the curves to one of the three groups.

\section{Fecundity experiments}

A total of 15 EG, iES and cES 1-2-day-old adult female wasps were individually paired for $24 \mathrm{~h}$ in small vials with a conspecific male. Females were then placed individually in arenas with leaf disks bearing $\sim 80$ whitefly nymphs. The leaf disks rested on a layer of agar in ventilated $35 \mathrm{~mm}$ agar Petri dishes. Females were transferred to new whitefly-infested arenas every other day until day 8 , as previous studies have shown the majority of reproduction occurs in this period (Vet and Van Lenteren, 1981). At day 4, we provided females with the opportunity to mate again to avoid the risk of sperm depletion by introducing a male into the arena for $24 \mathrm{~h}$. After females were removed on day 8 , arenas were incubated for $\sim 10$ more days. Arenas were kept at $27^{\circ} \mathrm{C}, 16 \mathrm{~h}$ light $/ 8 \mathrm{~h}$ dark and $65 \%$ relative humidity throughout the experiment. At the end of this period, we recorded the number of progeny pupae, a proxy for adult emergence, as a measure of fecundity. The cumulative number of progeny on days 2, 4, 6 and 8 of the oviposition period was compared among EG, iES and cES. Females were replaced only if they died within the first 2 days. Females that did not produce any progeny were considered unmated, and discarded. Only female progeny were produced in this experiment, as only whitefly hosts for female eggs were provided.

The comparison of fecundity of iES and cES in the experiment described above showed no apparent fecundity cost to bearing the Cardinium infection (see Results), in contrast to previous work with the same laboratory cultures (Perlman et al., 2008; Harris et al., 2010). Given the different results of the current study relative to published results, we tested the role of the laboratory environment in March 2016 by comparing fecundity differences of iES and cES in culture since 2003 with Cardinium-infected and antibiotic-cured wasps from 
additional ES cultures established from wasps collected from the same location of the old culture (Weslaco, TX, USA) in the summer of 2015. We replicated the experimental design described above, but in this second experiment we used 12 females from each of the 4 populations: iES and cES from the 2003 laboratory culture (Perlman et al., 2008; Harris et al., 2010); iESw ('wild'); collected from the field and in culture for $\sim 12$ generations before the experiment; and cESw. The infection status of $\mathrm{iESw}$ was confirmed with diagnostic PCR (Harris et al., 2010). The cured cESw wasps used to check the fecundity were produced by antibiotic curing of the Cardinium infection of their iESw grandparents $\left(50 \mathrm{mg} \mathrm{ml}^{-1}\right.$ rifampicin in honey for $48 \mathrm{~h}$ ), followed by no treatment of their parents. The success of curing was similarly confirmed by diagnostic PCR.

For analysis of both fecundity experiments, in which the number of offspring was counted at different time intervals, a linear mixed model with time and wasp identity as fixed effects and female as a random effect was built using the $\mathrm{R}$ package lme4 (Bates et al., 2013). For the second experiment comparing a longstanding laboratory culture with laboratory cultures recently established from the field, an additional linear model was built, this time using the total number of offspring by each female as the response variable, and including infection status (infected versus cured) and age of the culture (old versus young) and the interaction of these factors as explanatory variables. Visual inspection of residual plots did not reveal any deviations from homoscedasticity or normality.

\section{Longevity test}

The proportion of parental and hybrid wasps recovered from the population cages could not only reflect the rates of production of hybrids vs parental species wasps, but also be influenced by differential longevity of the different types of wasps. In particular, if there were differences in vigour between hybrid and parental type wasps, longevity differences could also influence recovery rates of adults of each type from the emergence jars. To test the latter hypothesis, we examined the relative longevity of parental species vs hybrid wasps. We isolated 20 female pupae of EG, $\mathrm{EES}, \mathrm{cES}$ and hybrids (produced by mating EG and cES wasps in both directions) in $1.2 \mathrm{ml}$ vials, and at emergence we fed the adults with a small drop of honey, and kept them at a constant temperature of $27^{\circ} \mathrm{C}$. Vials were checked daily, honey was added when necessary, and the date of female death was recorded. Data were analysed by analysis of variance (ANOVA) using $\mathrm{R}$.

\section{Model of cage population dynamics}

We developed a deterministic mathematical model that used empirically determined values of wasp fecundity, mate discrimination and hybrid viability and sterility to predict competitive outcomes in the two treatments. The model describes cage population dynamics in terms of the relative frequencies of the three types of wasps (cES, iES and EG) and contains all experimental twopopulation situations as special cases. In Figure 1, we depict the rationale behind our model. A random female (that is, of any of the three types) has probability $p_{m}$ of encountering a male of type $m$, respectively. We assume random encounters, so that these probabilities are identical to the relative frequencies at which the types occur in the cage. With probability $d_{f, m}$, a female of type $f$ discriminates against the encountered male type $m$ and does not mate (but may go on to encounter other males in the future). With probability $1-d_{f, m}$ she mates with the encountered male. Note that discrimination rates depend on both the male and female types. Census is made at the egg stage and fitness $(w)$ is fecundity, that is, the number of non-hybrid eggs, corrected for possible CI effects. Subscripts in fitness values depict the female type (except for $w_{\mathrm{CES}, \mathrm{iES}}$ and $w_{\mathrm{iES}, \mathrm{CES}}$ where the first subscript denotes the female and the second subscript denotes the male type). Fitness of interspecific matings is considered to be zero, given hybrid sterility. In total, there are nine fitness values and nine discrimination rates, corresponding to the number of possible crosses (Table 1). Fitness values are the 2-day average cumulative number of viable progeny produced over 8 days from the first fecundity experiment (see Results), whereas discrimination rates were calculated from Gebiola et al. (2016). Although Gebiola et al. (2016) assessed prezygotic isolation through a number of mating tests (no choice, female choice, male choice, multiple choice), we calculated discrimination rates from the female choice tests because, in most species, females are the sex that discriminates among males, as predicted by sexual selection theory. Based on Figure 1, we derived the following Equations $(1,2,3)$ that describe, for all three types of wasps (cES, iES or EG), how the number of viable, non-hybrid individuals of a given type changes from one generation to the next:

$$
\begin{aligned}
& c E S^{\prime}=\left(c E S * p_{\mathrm{cES}} *\left(1-d_{\mathrm{cES}, \mathrm{cES}}\right) * w_{\mathrm{cES}}+c E S * p_{\mathrm{iES}} *\left(1-d_{\mathrm{cES}, \mathrm{EES}}\right) * w_{\mathrm{cES}, \mathrm{iES}}\right) \\
& \sum_{j=0}^{n}\left(p_{\mathrm{cES}} * d_{\mathrm{cES}, \mathrm{CES}}+p_{\mathrm{iES}} * d_{\mathrm{cES}, \mathrm{ES}}+p_{\mathrm{EG}} * d_{\mathrm{cES}, \mathrm{EG}}\right)^{j} \\
& i E S^{\prime}=\left(i E S * p_{\mathrm{cES}} *\left(1-d_{\mathrm{iES}, \mathrm{cES}}\right) * w_{\mathrm{iES}, \mathrm{cES}}+i E S * p_{\mathrm{iES}} *\left(1-d_{\mathrm{iES}, \mathrm{iES}}\right) * w_{\mathrm{iES}}\right) \\
& \sum_{j=0}^{n}\left(p_{\mathrm{cES}} * d_{\mathrm{iES}, \mathrm{ES}}+p_{\mathrm{iES}} * d_{\mathrm{iES}, \mathrm{ES}}+p_{\mathrm{EG}} * d_{\mathrm{iES}, \mathrm{EG}}\right)^{j} \\
& E G^{\prime}=E G * p_{\mathrm{EG}} *\left(1-d_{\mathrm{EG}, \mathrm{EG}}\right) * w_{\mathrm{EG}} \\
& * \sum_{j=0}^{n}\left(p_{\mathrm{cES}} * d_{\mathrm{EG}, \mathrm{ESS}}+p_{\mathrm{iES}} * d_{\mathrm{EG}, \mathrm{ES}}+p_{\mathrm{EG}} * d_{\mathrm{EG}, \mathrm{EG}}\right)^{j}
\end{aligned}
$$

The summation terms $(\Sigma \ldots)$ take into account that a female might discriminate against several encountered males before finally mating. Because the chance of not encountering a suitable male gets smaller with each round, we chose $n=8$ as a maximum number of encounters (the average difference between 8 and 9 rounds results in $<1 \%$ change in terms of fecundity). In the model we included as an additional intergenerational step the random sampling of 80 eggs that start the new cage generation. Both the sex ratio and the relative frequencies of wasp types in the new generation were calculated according to the sex ratio and the relative frequencies of types among these 80 eggs. This second step mimics the experimental procedure and is the only stochastic element in our otherwise deterministic model. Mathematical results were averaged over 1000 runs. We used Python (version 2.7.11) for numerical analysis.

\section{RESULTS}

\section{Population cages}

The HRM assay was an effective tool for separating adult wasps into parental and hybrid categories. The assay revealed three nonoverlapping melting curves that corresponded to the three wasp

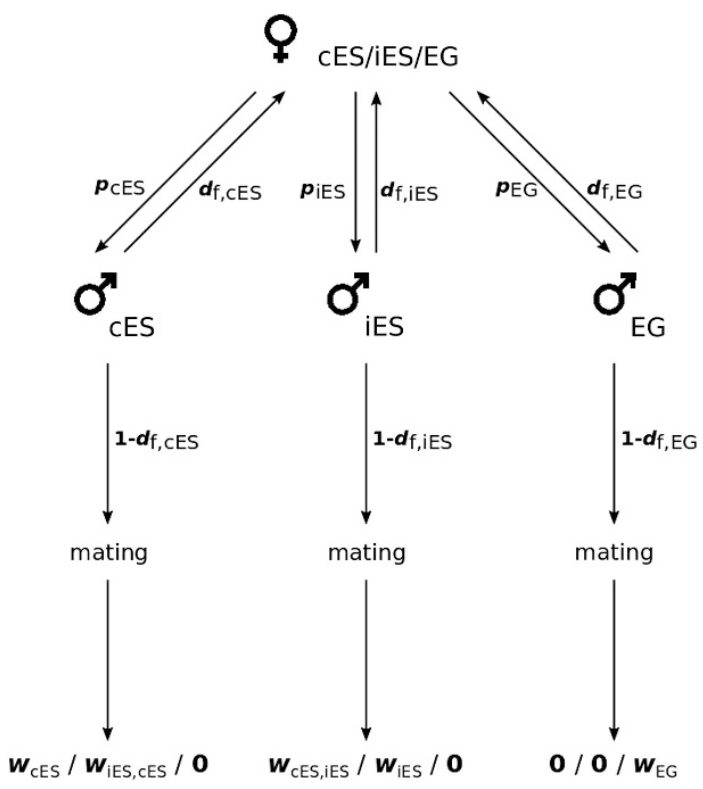

Figure 1 Possible matings in a cage containing all three types (cES, iES and EG) and the corresponding fitness outcomes. $d_{f, m}=$ probability of female type $f$ discriminating against male type $m ; p_{m}=$ probability of females encountering a male of type $m ; w=$ fitness (fecundity); $0=$ fitness of hybrid progeny. Further details are given in the main text. 
groups. The hybrid curves were different not only because their peaks were intermediate in location and height to those for the two parental species but also because hybrid curves had a different shape (Supplementary Figure S1).

Contrary to our predictions, treatment did not influence the outcome of competition in the population cages (Figures 2 and 3). In both treatments, E. suzannae, either $\mathrm{iES}$ or cES, outcompeted EG in three out of the four cages, with iES and cES excluding EG in two (by F3 and F5 generation) and three cages (by F4 in two cages and by F5 generation in the third), respectively. Interestingly, in one cage of each treatment, the outcome was reversed, with EG outcompeting, yet not

Table 1 Model parameters

\begin{tabular}{lcc}
\hline Cross $(F \times M)$ & Pupal progeny (not hybrid) & Discrimination rates \\
\hline cES $\times$ iES & 8.1 & 0.70 \\
$c E S \times E G$ & 0 & 0.85 \\
$i E S \times c E S$ & 29.2 & 0.60 \\
$i E S \times E G$ & 0 & 0.90 \\
$E G \times c E S$ & 0 & 0.51 \\
$E G \times i E S$ & 0 & 0.73 \\
$c E S \times c E S$ & 29.9 & 0.35 \\
ES $\times$ iES & 29.2 & 0.45 \\
$E G \times E G$ & 24.2 & 0.48 \\
\hline
\end{tabular}

Abbreviations: cES, cured Encarsia suzannae; EG, Encarsia gennaro; F, female; iES, infected Encarsia suzannae; M, male.

Fecundity values (pupal progeny) are the two-day average cumulative number of viable progeny produced over 8 days from the first fecundity experiment (see Results), whereas discrimination rates ( $=1-$ frequency of conspecific and heterospecific matings for intraspecific and interspecific crosses, respectively) were calculated from Gebiola et al. (2016). excluding, iES or cES. In cage 2 (EG-cES treatment), we ran the experiment for an extra generation, because it was not clear which species dominated at F5. By generation F6 EG dominated cES in this cage.

\section{Fecundity tests}

There were no significant differences in fecundity among wasp populations. In the first fecundity experiment, the average cumulative number of progeny produced over 8 days was 97.2 ( \pm 10.41 s.e.) for EG, 117.6 ( \pm 13.46 s.e.) for $\mathrm{iES}$ and 124.2 ( \pm 6.41 s.e.) for cES. In the second fecundity experiment, the average cumulative number of pupae produced over 8 days was 133.8 ( \pm 9.55 s.e.) for $\mathrm{iES}$ and 127.9 ( \pm 6.31 s.e.) for cES (both of these the long established laboratory cultures). Similarly, the cumulative number of pupal offspring for the recently collected laboratory cultures was 129.5 ( \pm 6.47 s.e.) for $\mathrm{iESw}$ and 121.7 ( \pm 6.18 s.e.) for $\mathrm{cESw}$. For both fecundity experiments, a linear mixed model with time and treatment as fixed effects and female as a random effect was a significantly better fit than a linear model (first experiment: likelihood ratio test $=37.922$, $P<0.01$; second experiment: likelihood ratio test $=8.944, P=0.001$ ). The mixed model for the first experiment showed a significant effect of time on wasp fecundity (ANOVA test, $\mathrm{F}=19.4, P<0.01$, d.f. $=3$ ), with a significant decrease at days 6 and 8 , whereas population identity did not have any effect $(F=1.9, P=0.168$, d.f. $=2$; Figure $4 a)$. The mixed model for the second experiment also showed a significant effect of time on fecundity (ANOVA test, $\mathrm{F}=20.1, P<0.01$, d.f. $=3$ ), with Tukey's post hoc test showing a significant decrease at days 4 and 6 , and a further decrease at day 8 , and again wasp identity was not significant $(F=0.9, P=0.440$, d.f. $=3$; Figure $4 \mathrm{~b})$. The linear model similarly did not find any significant effect of infection status or time

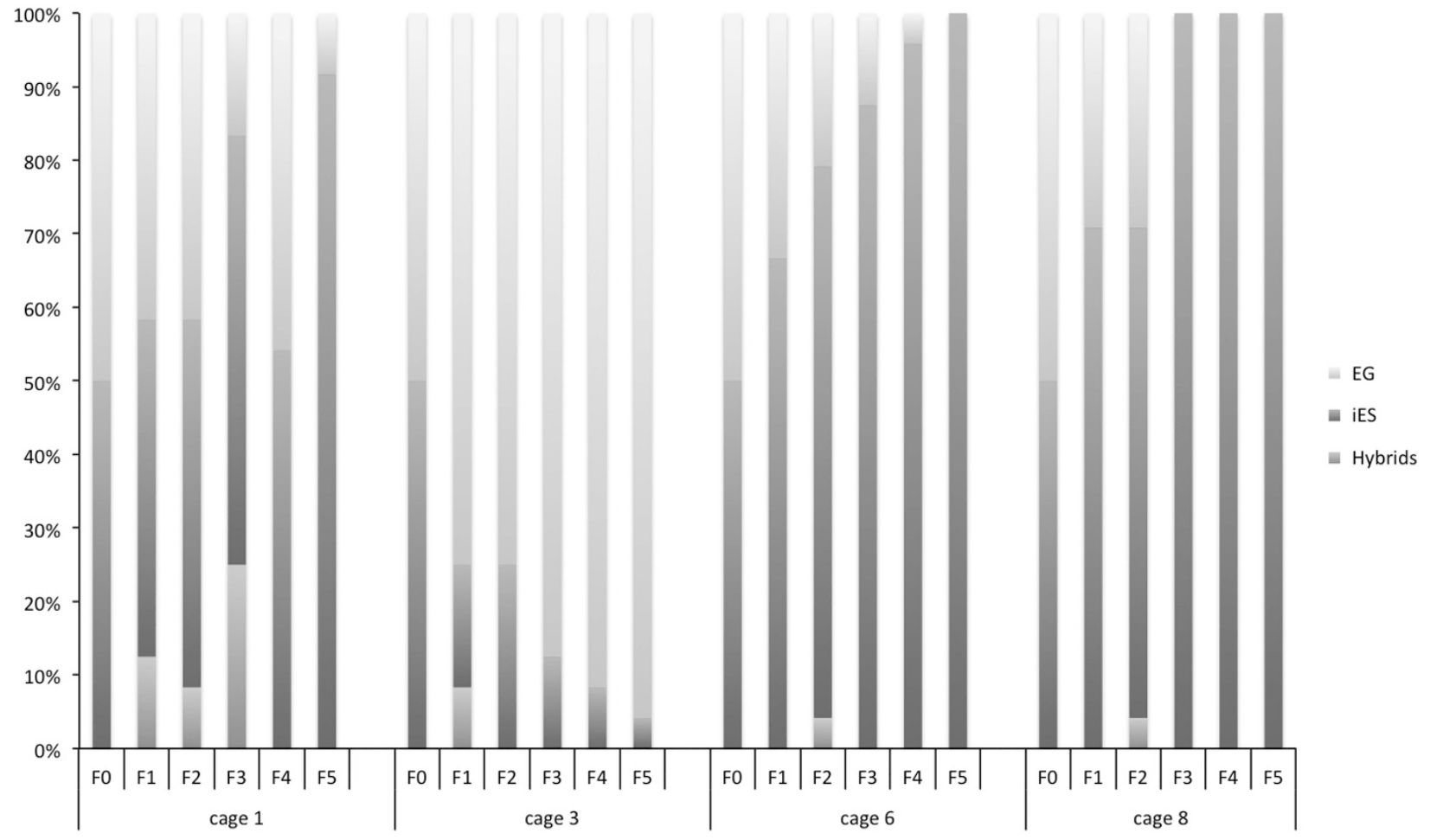

Figure 2 Stacked histogram summarizing the results of the competition between Encarsia gennaroi (EG) and infected E. suzannae iES. The $y$ axis represents the percentage of female progeny of EG, iES and hybrids as determined in each generation by HRM. Each bar represents 24 randomly selected genotyped females. 


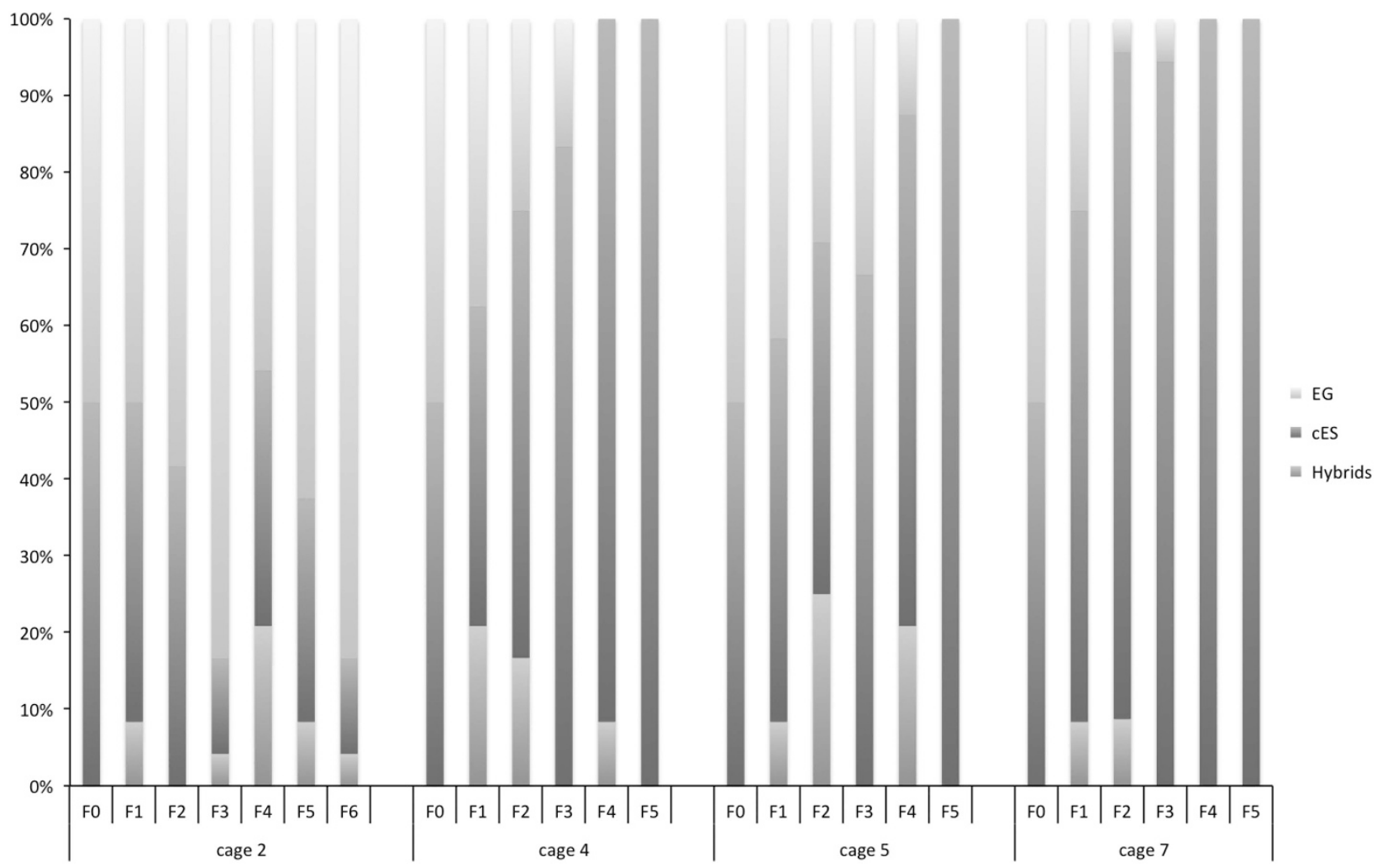

Figure 3 Stacked histogram summarizing the results of the competition between Encarsia gennaroi (EG) and cured E. suzannae (cES). The $y$ axis represents the percentage of female progeny of EG, cES and hybrids as determined in each generation by HRM. Each bar represents 24 randomly selected genotyped females.
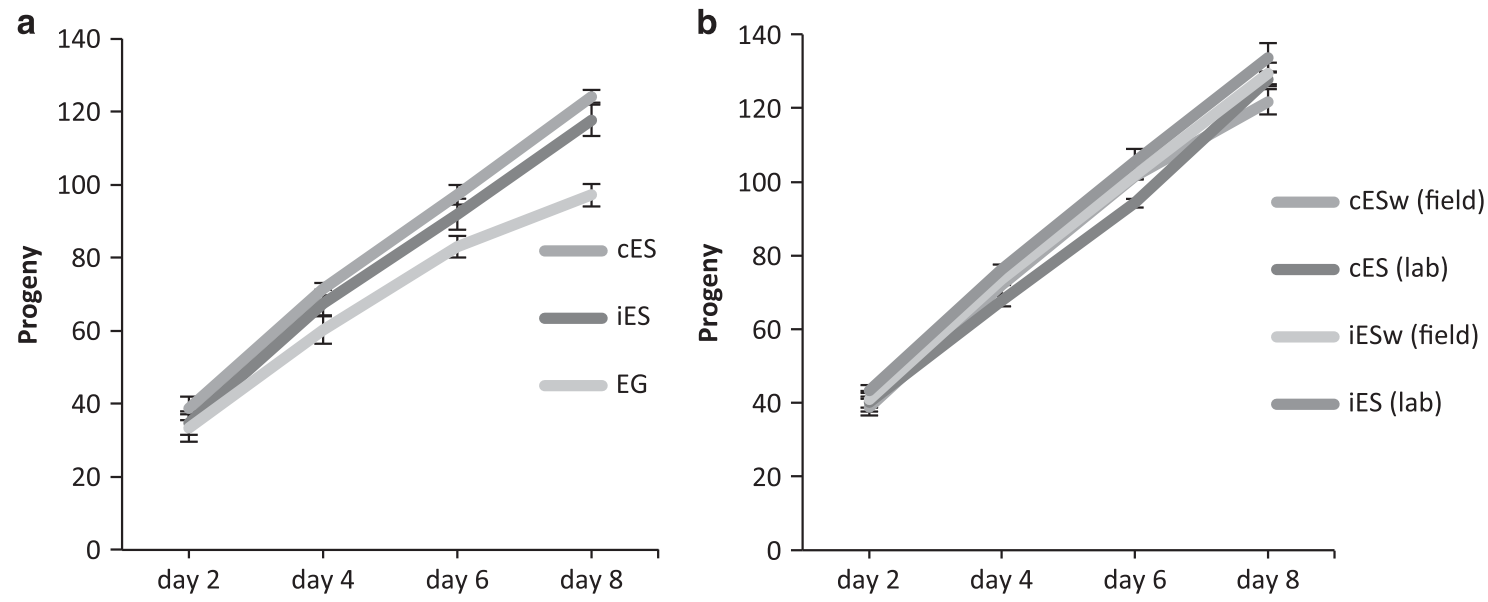

Figure 4 Mean number of cumulative offspring produced after 2, 4, 6 and 8 days by: (a) EG, iES and cES (first experiment); and (b) iES, cES, iESw and cESW (second experiment). Bars indicate s.e.

in culture as factors either as main effects or in interaction $(\mathrm{F}=0.97$, d.f. $=1, P=0.333$ for infection status; $\mathrm{F}=0.532$, d.f. $=1, P=0.471$ for time in culture; $\mathrm{F}=0.017$, d.f. $=1, P=0.898$ for their interaction).

\section{Longevity test}

On average, EG adults lived for 26.3 ( \pm 0.98 s.e.) days, $\mathrm{iES}$ for 27.9 ( \pm 0.82 s.e.) days, cES for 25.5 ( \pm 1.13 s.e.) days and hybrids for 26.3 $( \pm 0.84$ s.e.) days. No statistically significant difference in longevity was observed among the four groups (ANOVA test, $\mathrm{F}_{3,76}=1.15$,
$P=0.333$ ), illustrating that hybrids lived as long as parentals and were as likely to be sampled from the pools of adults produced in each generation.

\section{Theoretical versus experimental results}

The model predicted negative population growth for EG in all cages and ES dominance. In contrast to this prediction, two of the eight population cages showed competitive dominance of EG (one cage in each treatment). While acknowledging this qualitative discrepancy, we 
used the six remaining experimental cage results for quantitative comparisons between theory and experiment. Moreover, as the model does not consider hybrids, the latter were excluded from the population cage results and the relative frequencies of EG and ES were recalculated. Without any parameter fitting, the model describes these (averaged) results reasonably well (Figure 5). The fit between model and population cage results suggests that the model's key parameters (fecundity, discrimination rate) are also likely to be the most important parameters in the interactions of the wasp types.

\section{DISCUSSION}

In general, the type of role that CI symbionts play in competitive outcomes between sibling species might hinge on the strength of prezygotic and postzygotic barriers. If they are both weak, we might expect CI would lead to competitive dominance of the infected population. If postzygotic barriers are strong, as in the study system here, then life-history differences should influence the outcome, and here symbiont infection-associated fecundity costs might be important. We did not find any difference between the two population cage treatments (EG vs iES and EG vs cES), as ES (E. suzannae), whether iES or cES, displaced EG (E. gennaroi) in three out of four cages of each treatment. On one hand, this result supports our prediction that the direct effects of CI would be insignificant. In this system, CI-caused lethality in the EG-iES is replaced by hybrid sterility in the EG-cES treatment (Gebiola et al., 2016), and the generation of a greater number of sterile hybrid females in the EG-cES treatment would be unlikely to cause reproductive interference, as hybrids are only females, and unlikely to reduce mating opportunities of parental type males that mate multiply. On the other hand, though, the lack of difference between treatments was unforeseen because we expected the fecundity costs of bearing the CI Cardinium to be significant for the iES population (Perlman et al., 2008; Harris et al., 2010).

Although we predicted that the fecundity cost known to be sustained by E. suzannae (Perlman et al., 2008) would be important in determining competitive outcome in our system, we saw no evidence of such an effect in the population cages. This led us to reassess the fecundity cost associated with Cardinium infection. In a first experiment, we did not find any fecundity difference between infected and cured laboratory lines, leading us to hypothesize that adaptation to the laboratory conditions might have reduced the fecundity cost to Cardinium infection. To test this idea, we then carried out a second experiment, this time comparing the infected and cured laboratory lines with infected and cured lines recently established from infected specimens of E. suzannae collected in the field. This experiment showed that there was no difference in fecundity between cured and infected wasps in long-term laboratory culture as well as in culture established from the field within a year, and hence we can rule out 'laboratory adaptation' as a factor. There are two possible explanations for the discrepancy between our results and the results by Perlman et al. (2008). First, the original experiment was extraordinary in some way, and overestimated the fecundity costs due to symbiont infection. This is plausible given that the subsequent population cage experiments, which tested different initial infection frequencies to determine the conditions in which CI Cardinium would invade, showed invasion from even low frequencies of infection, suggesting only mild or nonexistent fecundity costs (Harris et al., 2010). Alternatively, in the 10 years since the earlier experiments were conducted ( $\sim 170$ generations), the Cardinium-E. suzannae association might have evolved to lessen fecundity costs in both laboratory and field populations. This is consistent with a finding of Weeks et al. (2007), who reported a striking example of a CI-Wolbachia infection in Drosophila simulans that went from imposing a $20 \%$ fecundity cost on its host to conferring a $10 \%$ fecundity advantage over uninfected females in $<20$ years ( $\sim 200$ generations), thus rapidly evolving toward commensalism or mutualism.

Having ruled out any fecundity cost associated with Cardinium, it is likely that the outcome of the competition between the two sibling species was driven by a few slight competitive advantages leading to reproductive interference. Population cages are often more sensitive assays than comparisons of performance parameters of different populations in isolation, because individuals of competing groups interact directly (Xi et al., 2005; Harris et al., 2010). However, in a situation like the one we examined, where hybrid matings occur, we expect that once one species became more frequent, reproductive interference would have ensured that species' eventual dominance (the dominance of E. suzannae over E. gennaroi in our cage system).
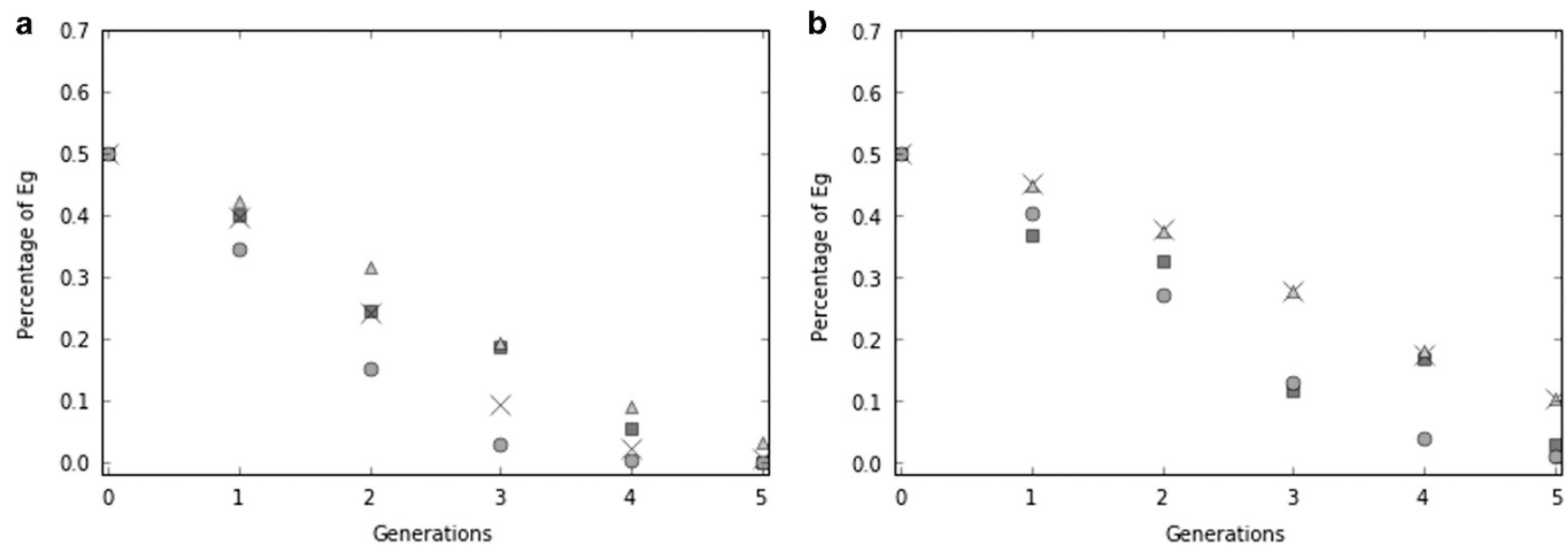

Figure 5 The decline of E. gennaroi (EG) when competing against E. suzannae, infected (iES) or cured (cES), as predicted by the deterministic model. The graphs show the frequency of EG over five generations in two different two-population settings: in competition with cES (a) and with iES (b). Shown are results from the experiment (green squares) and those obtained by the model (red circles). Moreover, model predictions are shown when fecundity values of both species are equalized (that is, set to the higher value, blue crosses) or discrimination rates are equalized (yellow triangles). Equalization of one parameter shows the influence of the other. A full colour version of this figure is available at the Heredity journal online. 
Consequences of reproductive interference are often frequency dependent, with the rarer species having greater difficulty finding conspecific mates. The effect of interspecific sexual interaction on population dynamics has long been overlooked, because often species are seen as discrete units of reproduction (Mallet, 2005; Kishi et al., 2009). Recently, increasing numbers of studies have documented reproductive interference between closely related species of various taxa (reviewed by Gröning and Hochkirch, 2008). Our model, which includes reproductive interference (that is, asymmetric mating preferences), fits the results we obtained in cage experiments well, suggesting that reproductive interference is important in this study system, and that it can be as influential as large differences in fecundity. Over a longer time span, reproductive interference often promotes niche segregation (spatial, temporal or habitat segregation) of closely related interacting species in nature (Gröning et al., 2007).

Asymmetries in mate choice can substantially contribute to the evolution of species-specific mate discrimination between closely related sympatric species, ultimately leading to divergence of the species recognition systems, assortative mating and the evolution of premating barriers via reinforcement (if speciation is incomplete and hybrids have low but non-zero fitness) or reproductive character displacement (RCD, if postzygotic isolation is complete) (Butlin, 1987; Grether et al., 2009). This may have also been the case for our system in nature. There is preliminary evidence of greater prezygotic barriers between E. gennaroi and E. suzannae in sympatry ((in Johnson (1996) as E. pergandiella light and dark form, see also Gebiola et al. (2016)). The crosses in Johnson (1996) showed that only 10\% of females in both directions produced any progeny, indicating that most of them did not mate at all. Furthermore, the contrast between the symmetry in prezygotic isolation between sympatric populations recorded by Johnson (1996) and the asymmetry recorded in our laboratory crosses and population cages could be explained by the fact that we crossed E. gennaroi from a region of allopatry (no selection for RCD) with E. suzannae from a region of sympatry (possible selection for RCD). Prezygotic isolation between closely related species can become weak or lost after prolonged periods of allopatry, because selection to maintain isolation is no longer present (Wellenreuther et al., 2010). A study like this one affords an opportunity to explore the processes that underlie outcomes that may be difficult to observe in the field. Indeed, the effect of reproductive interference on population persistence is generally expected to be evolutionarily short-lived because of what may be rapid evolution of RCD (Kyogoku, 2015). Our results are at least consistent, although indirectly, with the evolution of RCD by E. suzannae.

Both our population cage results and our model may also reflect the range of outcomes in the field. The two species still coexist in Southern Texas as of 2015. A sampling from March 2003 indicated that E. gennaroi was the dominant species at the time, whereas E. suzannae was prevalent in July 2015 (our unpublished data). Given these fluctuations in abundance, it is not clear whether this coexistence is long-lived, but it is interesting that it has persisted for two decades at the very least. Although E. suzannae appeared to dominate the competitive interaction, the fact that $25 \%$ of the cages were dominated by E. gennaroi suggests that we might expect even more variation in outcomes in an environment more complex than a population cage. In the field, the frequency of heterospecific encounters can be substantially lower than in small arenas with unnaturally high abundance (Coyne et al., 2005; Bargielowski et al., 2015), and species might utilize different microhabitats and occur in different abundance or intraspecific aggregations, leading to small-scale segregation and a reduced frequency of encounters in nature (Hochkirch et al., 2007). As a result, competitive displacements are dynamic, ongoing and reversible processes, and competitive reductions (Lounibos, 2007) are more likely to occur than complete species exclusions (DeBach, 1966; Reitz and Trumble, 2002).

The possibility of coexistence is also hinted at in our model. We explored the behaviour of the outcomes of species interactions in the parameter space spanned by both heterospecific discrimination rates (E. gennaroi against E. suzannae and vice versa, both for infected and cured E. suzannae). We found that the stronger the nondominant species discriminates against the other species, the longer it takes for the nondominant species to go extinct (Supplementary Figure S2). Although the timeframe of coexistence is not very large (extinction occurs after 12 generations, at the latest), this might easily change if adaptive changes within species are taken into account (which is not the case in our model). This could, in principle, lead to long-term coexistence. Moreover, we find a narrow transition phase where the outcome of competitive interactions is reversed, that is, the previously dominant species becomes nondominant, and vice versa (Supplementary Figure S2). This might reflect reversals in dominance in the field.

In conclusion, interspecific interactions between incipient species are likely to have profound ecological and evolutionary consequences. CI symbionts may not be important, when, as in the present study, isolation is complete and there are no fecundity costs to CI symbiont infection. In general, however, CI symbionts may be important directly, when genetic isolation is not complete, or indirectly, when fecundity costs depress the competitiveness of one population. More important in our system, as our model predicted, weak prezygotic barriers of the type present in the E. gennaroi-E. suzannae interaction can lead to frequency-dependent reproductive interference and the rapid displacement of one population by the other. The model generally predicted the outcome we observed, although the cage experiment, in which E. gennaroi displaced E. suzannae in two of the eight cages, suggested a greater role of stochastic events in determining which of the species prevails. Somewhat speculatively, the cage experiments could relate to the history of interaction of these species in sympatry in South Texas, where there is some preliminary evidence of reversals in dominance but both species persist, perhaps long enough for reproductive character displacement to evolve and coexistence to be possible.

\section{DATA ACCESSIBILITY}

Fecundity, longevity and HRM data available from the Dryad Digital Repository: http://dx.doi.org/10.5061/dryad.8v739.

\section{CONFLICT OF INTEREST}

The authors declare no conflict of interest.

\section{ACKNOWLEDGEMENTS}

We are very grateful to Adrian Silva and Bill Warfield for collecting Encarsia suzannae from the Rio Grande Valley, Robert Palais for providing the program MW6 and for helping with its usage, Paul Rugman-Jones and Robert Palais for advising on HRM, and Dakota Maness, Rachel Wilson-King and Jimmy Conway for helping with the HRM assays. This research was supported by a Marie Curie International Outgoing Fellowship within the 7th European Community Framework Programme, Grant Agreement Number PIOFGA-2012-327425 to MG and MGi, and by the National Science Foundation Grant IOS-1256905 to MSH. 
de Almeida FB, Moura AS, Cardoso AF, Winter CE, Bijovsky AT, Suesdek L (2011). Effects of Wolbachia on fitness of Culex quinquefasciatus (Diptera; Culicidae). Infect Genet Evol 11: 2138-2143.

Bargielowski IE, Lounibos LP, Carrasquilla MC (2013). Evolution of resistance to satyrization through reproductive character displacement in populations of invasive dengue vectors. Proc Natl Acad Sci USA 110: 2888-2892.

Bargielowski IE, Lounibos LP, Shin D, Smartt CT, Carrasquilla MC, Henry A et al. (2015). Widespread evidence for interspecific mating between Aedes aegypti and Aedes albopictus (Diptera: Culicidae) in nature. Infect Genet Evol 36: 456-461.

Bates D, Maechler M, Bolker B, Walker S (2013). Ime4: Linear mixed-effects models using Eigen and S4. R package version 1.0-5. http://CRAN.R-project.org/package $=$ Ime4

Bordenstein SR (2003). Symbiosis and the origin of species. In: Bourtzis K, Miller TA. (eds). Insect Symbiosis Vol. 1, CRC Press: Florida. pp 283-304.

Butlin R (1987). Speciation by reinforcement. Trends Ecol Evol 2: 8-13.

Cothran RD (2015). The importance of reproductive interference in ecology and evolution: from organisms to communities. Popul Ecol 57: 339-341.

Coyne JA, Elwyn S, Rolán-Alvarez EL (2005). Impact of experimental design on Drosophila sexual isolation studies: direct effects and comparison to field hybridization data. Evolution 59: 2588-2601.

Coyne JA, Orr HA (2004). Speciation. Sinauer: Massachussets.

DeBach P (1966). The competitive displacement and coexistence principles. Annu Rev Entomol 11: 183-212.

Dwight Z, Palais R, Wittwer CT (2011). UMELT: prediction of high-resolution melting curves and dynamic melting profiles of PCR products in a rich web application. Bioinformatics 27: 1019-1020.

Egas M, Vala F, Breeuwer JAJ (2002). On the evolution of cytoplasmic incompatibility in haplodiploid species. Evolution 56: 1101-1109.

Gebiola M, Kelly SE, Hammerstein P, Giorgini M, Hunter MS (2016). “Darwin's corollary' and cytoplasmic incompatibility induced by Cardinium may contribute to speciation in Encarsia wasps (Hymenoptera: Aphelinidae). Evolution 70: 2447-2458.

Gebiola M, Monti MM, Johnson RC, Woolley JB, Hunter MS, Giorgini M et al. (2017). A revision of the Encarsia pergandiella species complex (Hymenoptera: Aphelinidae) shows cryptic diversity in parasitoids of whitefly pests. Syst Entomol 42 31-59.

Grether GF, Losin N, Anderson CN, Okamoto K (2009). The role of interspecific interference competition in character displacement and the evolution of competitor recognition. Biol Rev 84: 617-635.

Gröning J, Hochkirch A (2008). Reproductive interference between animal species. Quart Rev Biol 83: 257-281.

Gröning J, Lücke N, Finger A, Hochkirch A (2007). Reproductive interference in two ground-hopper species: testing hypotheses of coexistence in the field. Oikos 116: 1449-1460.

Harris LR, Kelly SE, Hunter MS, PerIman SJ (2010). Population dynamics and rapid spread of Cardinium, a bacterial endosymbiont causing cytoplasmic incompatibility in Encarsia pergandiella (Hymenoptera: Aphelinidae). Heredity 104: 239-246.

Hochkirch A, Gröning J, Bücker A (2007). Sympatry with the devil: reproductive interference could hamper species coexistence. J Anim Ecol 76: 633-642.

Hunter MS, Perlman SJ, Kelly SE (2003). A bacterial symbiont in the Bacteroidetes induces cytoplasmic incompatibility in the parasitoid wasp Encarsia pergandiella. Proc $R$ Soc B Biol Sci 270: 2185-2190.

Hunter MS, Woolley JB (2001). Evolution and behavioral ecology of heteronomous aphelinid parasitoids. Annu Rev Entomol 46: 251-290.
Johnson RC (1996). Biosystematics of Encarsia pergandiella and Encarsia tabacivora, two cryptic species of whitefly parasitoids (Hymenoptera: Aphelinidae). MSc thesis, Texas A\&M University, College Station, TX.

Kishi S, Nishida T, Tsubaki Y (2009). Reproductive interference determines persistence and exclusion in species interactions. J Anim Ecol 78: 1043-1049.

Kyogoku D (2015). Reproductive interference: ecological and evolutionary consequences of interspecific promiscuity. Pop Ecol 57: 253-260.

Li M, Zhou L, Palais RA, Wittwer CT (2014). Genotyping accuracy of high-resolution DNA melting instruments. Clin Chem 60: 864-872.

Liou LW, Price TD (1994). Speciation by reinforcement of premating isolation. Evolution 48: 1451-1459.

Liu S, De Barro P, Xu J, Luan J, Zang L, Ruan Y et al. (2007). Asymmetric mating interactions drive widespread invasion and displacement in a whitefly. Science 318: 1769-1772.

Lounibos LP (2007). Competitive displacement and reduction. J Am Mosquito Control Assoc 23: 276-282.

Mallet J (2005). Hybridization as an invasion of the genome. Trends Ecol Evol 20: 229-237.

Ord TJ, King L, Young AR (2011). Contrasting theory with the empirical data of species recognition. Evolution 65: 2572-2591.

Perlman SJ, Kelly SE, Hunter MS (2008). Population biology of cytoplasmic incompatibility: maintenance and spread of Cardinium symbionts in a parasitic wasp. Genetics 178: 1003-1011.

Perrot-Minnot M, Cheval B, Migeon A, Navajas M (2002). Contrasting effects of Wolbachia on cytoplasmic incompatibility and fecundity in the haplodiploid mite Tetranychus urticae. J Evol Biol 15: 808-817.

Reed GH, Kent KO, Wittwer CT (2007). High-resolution DNA melting analysis for simple and efficient molecular diagnostics. Pharmacogenomics 8: 597-608.

Reitz SR, Trumble JT (2002). Competitive displacement among insects and arachnids. Annu Rev Entomol 47: 435-465.

Rugman-Jones PF, Stouthamer R (2016). High resolution melt analysis without DNA extraction affords rapid genotype resolution and species identification. Mol Ecol Res 17: 598-607.

Servedio M, Noor MAF (2003). The role of reinforcement in speciation: theory and data. Annu Rev Ecol Syst 34: 339-364.

Untergasser A, Cutcutache I, Koressaar T, Ye J, Faircloth BC, Remm M et al. (2012). Primer3-new capabilities and interfaces. Nucl Acids Res 40: e115.

Vet LEM, van Lenteren JC (1981). A comparison of three Encarsia species and one Eretmocerus species to estimate their potentialities in controlling whitefly in tomatoes in greenhouses with a low temperature regime. J Appl Entomol 91: 327-348.

Weeks AR, Turelli M, Harcombe WR, Reynolds KT, Hoffmann AA (2007). From parasite to mutualist: rapid evolution of Wolbachia in natural populations of Drosophila. PLoS Biol 5: e114.

Wellenreuther M, Tynkkynen K, Svensson EI (2010). Simulating range expansion: male species recognition and loss of premating isolation in damselflies. Evolution 64: 242-252.

Werren JH, Baldo L, Clark ME (2008). Wolbachia: master manipulators of invertebrate biology. Nat Rev Microbiol 6: 741-751.

Wong WH, Tay YC, Puniamoorthy J, Balke M, Cranston PS, Meier R (2014). 'Direct PCR' optimization yields a rapid, cost-effective, nondestructive and efficient method for obtaining DNA barcodes without DNA extraction. Mol Ecol Res 14: 1271-1280.

Xi ZY, Khoo CCH, Dobson SL (2005). Wolbachia establishment and invasion in an Aedes albopictus laboratory population. Science 310: 326-328.

Supplementary Information accompanies this paper on Heredity website (http://www.nature.com/hdy) 